\title{
Comprehensive analysis of key genes and microRNAs in radioresistant nasopharyngeal carcinoma
}

\author{
Ya Guo ${ }^{1 *}$, Yang Zhang ${ }^{1}$, Shu Juan Zhang ${ }^{2}$, Yi Nan Ma ${ }^{1}$ and Yun $\mathrm{He}^{1}$
}

\begin{abstract}
Background: Radioresistance is one of the main obstacle limiting the therapeutic efficacy and prognosis of patients, the molecular mechanisms of radioresistance is still unclear. The purpose of this study was to identify the key genes and miRNAs and to explore their potential molecular mechanisms in radioresistant nasopharyngeal carcinoma.

Methods: In this study, we analysis the differentially expressed genes and microRNA based on the database of GSE48501 and GSE48502, and then employed bioinformatics to analyze the pathways and GO terms in which DEGs and DEMS target genes are involved. Moreover, Construction of protein-protein interaction network and identification of hub genes. Finally, analyzed the biological networks for validated target gene of hub miRNAs.

Results: A total of 373 differentially expressed genes (DEGs) and 14 differentially expressed microRNAs (DEMs) were screened out. The up-regulated gene JUN was overlap both in DEGs and publicly available studies, which was potentially targeted by three miRNAs, including hsa-miR-203, hsa-miR-24 and hsa-miR-31. Moreover, Pathway analysis showed that both up-regulated gene and DEMs target genes were enriched in TGF-beta signaling pathway, Hepatitis B, Pathways in cancer and p53 signaling pathway. Finally, we further constructed protein-protein interaction network (PPI) of DEGs and analyzed the biological networks for above mentioned common miRNAs, the result indicated that JUN was a core hub gene in PPI network, hsa-miR-24 and its target gene were significantly enriched in P53 signaling pathway.
\end{abstract}

Conclusions: These results might provide new clues to improve the radiosensitivity of Nasopharyngeal Carcinoma.

Keywords: Nasopharyngeal carcinoma, microRNA, gene expression omnibus differentially expressed genes, bioinformatics analysis

\section{Background}

Radiotherapy is a mainly treatment for nasopharyngeal carcinoma (NPC). However, radioresistance is one of the major factors to affect the therapeutic efficacy and prognosis of patients [1-3]. Accordingly, identifying potential biomarkers and studying the molecular mechanisms associated with radioresistant nasopharyngeal carcinoma has become a hot topic both in basic and clinical research.

Microarrays are considered to be an important method for identifying potential biomarkers in many diseases at the molecular level with more effective and detailed insights [4]. Several microRNAs and mRNAs have been

\footnotetext{
* Correspondence: gy8569851@163.com

${ }^{1}$ Department of Oncology, The Second Affiliated Hospital of Medical College, Xi'an Jiao Tong University, 157 xi wu road, Xi'an 710004, People's Republic of China

Full list of author information is available at the end of the article
}

discovered to be involved in radioresistant NPC, whereas traditional methods have failed to elucidate the interaction of mRNAs and microRNAs and the molecular mechanisms of NPC due to the limitations on the comparative analysis [5-7]. Therefore, systematically investigating the interaction between microRNA and mRNA, and elucidating the molecular mechanism of radioresistant NPC is of great significance. With the development of bioinformatics, we can apply global analysis to process the data generated by microarray technology and find the interaction between DEGs and DEM, especially in the pathway interaction network, to summarize their potential mechanisms in diseases [8-10]. Based on above mentioned reasons, the present study aims to identify the key genes and miRNAs and to explore their potential molecular mechanisms in radioresistant nasopharyngeal carcinoma. 
In this study, we analysis the differentially expressed genes and microRNA between radioresistant NPC CNE2-R cells and radiosensitive CNE2 cells based on the database of GSE48501 and GSE48502, and then employed bioinformatics to analyze the pathways and GO terms in which DEGs and DEMS target genes are involved. Moreover, Construction of protein-protein interaction network and identification of hub genes. Finally, analyzed the biological networks for validated target gene of hub miRNAs. Our data may provide an important contribution to identified biological markers and clarify the mechanisms of NPC radioresistance.

\section{Results}

\section{DEGs and DEMs in radioresistant NPC cells compared} with radioresistant NPC cells

GEO2R analyzed result shown that a total of 373 DEGs were identified in radioresistant NPC cells, including 291 mRNAs were up-regulated and 82 mRNAs were down-regulated (Table 1). The DEMs results indicated that there were 277 miRNAs were detected, 14 of which were differentially expressed with $\geq 1.5$ fold-change (t-test, $P<0.05$ ), including 4 up-regulated miRNAs and 10 down-regulated miRNAs (Table 2). Moreover, DigSee software were used to identify the radioresistant related genes for publicly available studies, 37 related genes were retrieved. In addition, Venn diagram analyses revealed that JUN and SOD2 were common both in the DEGs and the DigSee (Fig. 1a). Furthermore, we identified JUN related microRNA by mirDIP software and analyzed the common microRNAs between the JUN-related microRNAs and DEMs by Venn diagram software. 35 JUN-related microRNA were retrieved, 3 down-regulated microRNAs were detected which were joint in JUN-related microRNAs and DEMs, including hsa-miR-203, hsa-miR-24 and hsa-miR-31 (Table 3 and Fig. 1b).

\section{Gene ontology analysis of DEMs target genes and DEGs}

We performed gene ontology (GO) analysis of DEGs and DEMs target genes. Our result indicated that the significantly enriched GO terms of up-regulated and down-regulated microRNAs target genes were mainly involved in mitotic cell cycle; RNA binding; nucleoplasm; cytosol; biosynthetic process; gene expression; cellular nitrogen compound metabolic process; ion binding (Table 4). As shown in Fig. 2, the most significantly enriched GO terms corresponded to up-regulated DEGs were "Anti-apoptosis" (Ontology Biological Process), the most significant biological process for the down-regulated genes are Chromosome organization and biogenesis.

\section{Pathway enrichment analyses}

We performed pathways enrichment analysis of DEMs target genes and DEGs using DIANA miRPATH tool and

Table 1 Differential mRNA expression profile of radioresistant nasopharyngeal carcinoma CNE2R versus CNE-2 cells (The Table 1 show the top 20 differential expression genes)

\begin{tabular}{|c|c|c|}
\hline Gene Symbol & Description & Fold Change \\
\hline LXN & latexin & 22.53 \\
\hline IGFBP3 & insulin-like growth factor binding protein 3 & 18.88 \\
\hline$A B C G 1$ & ATP-binding cassette, sub-family G (WHITE), member 1 & 16.82 \\
\hline $\mathrm{CP}$ & ceruloplasmin (ferroxidase) & 14.76 \\
\hline TRIM31 & tripartite motif-containing 31 & 12.30 \\
\hline NNMT & nicotinamide $\mathrm{N}$-methyltransferase & 10.96 \\
\hline GDF15 & growth differentiation factor 15 & 10.15 \\
\hline INHBE & inhibin, beta $\mathrm{E}$ & 9.59 \\
\hline EGR1 & early growth response 1 & 7.95 \\
\hline IL8 & interleukin 8 & 7.49 \\
\hline METTL7A & methyltransferase like $7 \mathrm{~A}$ & 7.31 \\
\hline LOC387763 & hypothetical LOC387763 & 7.24 \\
\hline LCN2 & lipocalin 2 & 6.82 \\
\hline EDN2 & endothelin 2 & 6.57 \\
\hline BMP2 & bone morphogenetic protein 2 & 6.56 \\
\hline C8orf4 & chromosome 8 open reading frame 4 & 6.42 \\
\hline ASNS & asparagine synthetase & 6.12 \\
\hline SLC16A6 & solute carrier family 16, member 6 (monocarboxylic acid transporter 7) & 5.55 \\
\hline PCK2 & phosphoenolpyruvate carboxykinase 2 (mitochondrial) & 5.44 \\
\hline STEAP4 & STEAP family member 4 & 5.32 \\
\hline
\end{tabular}


Table 2 Differentially expressed miRNAs in GSE48502

\begin{tabular}{lll}
\hline miRNA & Fold change & P-value \\
\hline $\begin{array}{ll}\text { Up-regulated miRNA } \\
\text { hsa-miR-762 }\end{array}$ & 2.510 & 0.00337 \\
hsa-miR-1202 & 2.292 & 0.0008 \\
hsa-miR-193b & 1.530 & 0.00986 \\
hsa-let-7e & 1.521 & 0.00054 \\
Down-regulated miRNA & & \\
hsa-miR-203 & 3.337 & 0.01698 \\
hsa-miR-545 & 1.980 & 0.04888 \\
hsa-miR-4291 & 1.722 & 0.00271 \\
hsa-miR-183 & 1.677 & 0.03486 \\
hsa-miR-24 & 1.667 & 0.00032 \\
hsa-miR-130a & 1.598 & 0.01252 \\
hsa-miR-660 & 1.578 & 0.01531 \\
hsa-miR-31 & 1.535 & 0.00208 \\
hsa-miR-23a & 1.527 & 0.03552 \\
hsa-miR-30a & 1.526 & 0.0274 \\
\hline
\end{tabular}

Clue go software respectively. For the pathway analysis, the top of 30 significant pathways were selected in the DEMs target genes (Fig. 3a). Moreover, the upregulated DEGs were enriched in $12 \mathrm{kegg}$ pathways (Fig. 3b). We further identified 4 significant pathways both in up-regulated DEGs and DEMs, including TGF-beta signaling pathway, Hepatitis B, Pathways in cancer and p53 signaling pathway which were considered as crucial pathways (Fig. 3c). Finally, we analyzed the above mentioned microRNAs (hsa-miR-203, hsa-miR-24 and hsa-miR-31) and their corresponding pathways using Clue go and Clue pedia software. The results indicated that hsa-miR-24 and 20 target genes were associated with 7 pathways, and the P53 signaling pathway is the most significant pathway (Fig. 3d). Our result indicated that
P53 signaling pathway may be related to nasopharyngeal carcinoma radioresistance.

\section{Protein-protein interaction network and subnetwork of DEGs}

The PPI network was consisted of 339 nodes and 714 edges which were mapped by STRING software (Fig. 4). As shown in Fig. 5, the PPI network of DEGs was composed of 72 nodes that were interacted with each other. The connectivity degree of each node was calculated in this PPI network and the top 5 nodes with degree more than 20 were JUN, FOS, IL8, EGR, HSPA8. Among these genes, JUN with highest degree (54) in the Protein-Protein interaction network was considered as the hub node which have closely interacted with other genes. Therefore, we can infer that the up-regulated JUN may be a key node related with radioresistant nasopharyngeal carcinoma.

\section{Discussion}

Nasopharyngeal carcinoma is a geographically distributed disease, especially in southern china and southeast Asia. Although radiotherapy is considered to be the primary treatment for NPC, radioresistance-induced locoregional recurrence and distant metastases remains mainly obstacle to successful treatment $[11,12]$. Therefore, in-depth study biomarkers and mechanisms of radioresistance in NPC is of great significance for improving the radiosensitivity of NPC and provide a new ideas for design of good therapy.

Previous studies have identified that radioresistance-associated molecules (mRNAs, microRNAs, and proteins) regulate radioresistance through different biological process, such as DNA repair, apoptosis, cell cycle, and protective autophagy [9]. However, the molecular mechanisms underlying NPC radioresistnce remain elusive.

A

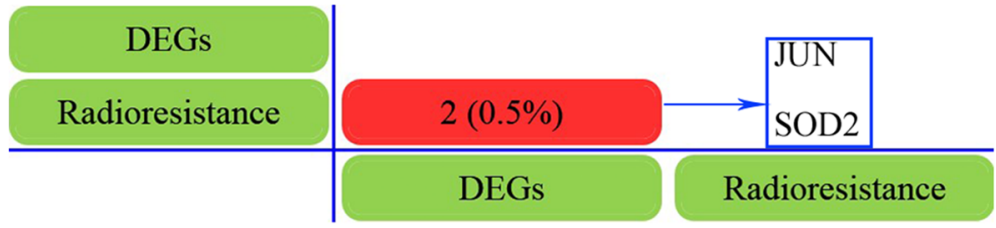

B

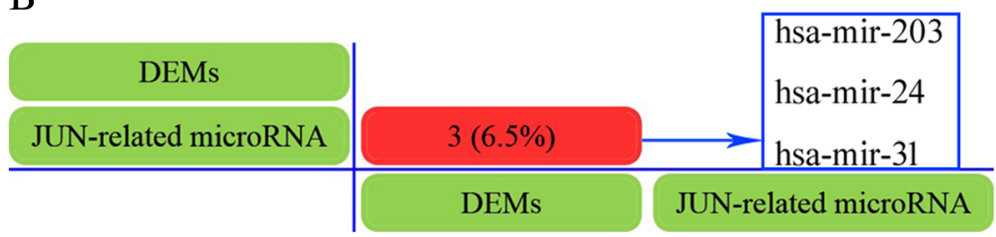

Fig. 1 Screening common genes or miRNAs by Venn diagram software. a Identification common genes between the DEGs and the publicly available studies by Venn diagram. b Analyzed the common microRNAs between the JUN-related microRNAs and DEMs by Venn diagram software 
Table 3 Identification of JUN related microRNA by mirDIP software. Prediction analysis was performed by mirDIP online software. In this table, asterisk represents common microRNA in DEMs and JUN-related microRNAs by Veen analysis

\begin{tabular}{|c|c|c|c|}
\hline Gene Symbol & MicroRNA & Integrated Score & Score Class \\
\hline JUN & hsa-miR-200b-3p & 0.8428 & Excellent \\
\hline JUN & hsa-miR-139-5p & 0.7769 & Excellent \\
\hline JUN & hsa-miR-200c-3p & 0.7693 & Excellent \\
\hline JUN & hsa-miR-429 & 0.7576 & Excellent \\
\hline JUN & hsa-miR-495-3p & 0.7162 & Excellent \\
\hline JUN & hsa-miR-32-5p & 0.6837 & Excellent \\
\hline JUN & hsa-miR-92a-3p & 0.6745 & Excellent \\
\hline JUN & hsa-miR-216b-5p & 0.6528 & Excellent \\
\hline JUN & hsa-miR-522-3p & 0.6392 & Excellent \\
\hline JUN & hsa-miR-501-5p & 0.60082 & Excellent \\
\hline JUN & hsa-miR-200a-3p & 0.5751 & Excellent \\
\hline JUN & hsa-miR-524-5p & 0.5637 & Excellent \\
\hline JUN & hsa-miR-520d-5p & 0.5365 & Excellent \\
\hline JUN & hsa-miR-141-3p & 0.5211 & Excellent \\
\hline JUN & hsa-miR-203* & 0.5019 & Excellent \\
\hline JUN & hsa-miR-580-3p & 0.4817 & Excellent \\
\hline JUN & hsa-miR-940 & 0.4770 & Excellent \\
\hline JUN & hsa-miR-1299 & 0.4628 & Excellent \\
\hline JUN & hsa-miR-9-5p & 0.4390 & Excellent \\
\hline JUN & hsa-miR-612 & 0.4313 & Excellent \\
\hline JUN & hsa-miR-583 & 0.4260 & Excellent \\
\hline JUN & hsa-miR-455-3p & 0.4018 & Excellent \\
\hline JUN & hsa-miR-637 & 0.3870 & Excellent \\
\hline JUN & hsa-miR-92b-3p & 0.3700 & Excellent \\
\hline JUN & hsa-miR-758-3p & 0.3659 & Excellent \\
\hline JUN & hsa-miR-25-3p & 0.3602 & Excellent \\
\hline JUN & hsa-miR-24* & 0.3585 & Excellent \\
\hline JUN & hsa-miR-31* & 0.3585 & Excellent \\
\hline JUN & hsa-miR-493-5p & 0.3318 & Excellent \\
\hline JUN & hsa-miR-127-5p & 0.3255 & Excellent \\
\hline JUN & hsa-miR-633 & 0.3227 & Excellent \\
\hline JUN & hsa-miR-766-3p & 0.3199 & Excellent \\
\hline JUN & hsa-miR-224-3p & 0.3097 & Excellent \\
\hline JUN & hsa-miR-494-3p & 0.3081 & Excellent \\
\hline JUN & hsa-miR-1285-3p & 0.3039 & Excellent \\
\hline
\end{tabular}

In order to understand the mechanisms underlying in radioresistant NPC, we performed global analysis of key genes and microRNAs in radioresistant Nasopharyngeal Carcinoma by bioinformatics analysis. Our result demonstrated that a total of 373 DEGs and 14 DEMs between radioresistant NPC CNE2-IR cells and radiosensitive CNE2 cells were identified. Our study revealed that JUN
Table 4 GO functional annotation of DEMs (Top 10)

\begin{tabular}{|c|c|c|c|}
\hline GO Category & Gene Target & miRNAs & P-value \\
\hline \multicolumn{4}{|l|}{ Up-regulated miRNAs } \\
\hline mitotic cell cycle & 158 & 4 & 0 \\
\hline $\begin{array}{l}\text { protein binding transcription } \\
\text { factor activity }\end{array}$ & 143 & 4 & 0 \\
\hline RNA binding & 473 & 4 & 0 \\
\hline nucleoplasm & 369 & 4 & 0 \\
\hline cytosol & 664 & 4 & 0 \\
\hline biosynthetic process & 909 & 4 & 0 \\
\hline gene expression & 234 & 4 & 0 \\
\hline viral process & 177 & 4 & 0 \\
\hline $\begin{array}{l}\text { cellular nitrogen compound } \\
\text { metabolic process }\end{array}$ & 1112 & 4 & 0 \\
\hline ion binding & 1145 & 4 & 0 \\
\hline \multicolumn{4}{|l|}{ Down-regulated miRNAs } \\
\hline nucleoplasm & 612 & 18 & 0 \\
\hline biosynthetic process & 1636 & 18 & 0 \\
\hline gene expression & 389 & 18 & 0 \\
\hline $\begin{array}{l}\text { cellular nitrogen compound } \\
\text { metabolic process }\end{array}$ & 2032 & 18 & 0 \\
\hline organelle & 3947 & 18 & 0 \\
\hline ion binding & 2198 & 16 & 0 \\
\hline mitotic cell cycle & 221 & 15 & 0 \\
\hline RNA binding & 812 & 15 & 0 \\
\hline $\begin{array}{l}\text { cellular protein modification } \\
\text { process }\end{array}$ & 985 & 15 & 0 \\
\hline cytosol & 1170 & 15 & 0 \\
\hline
\end{tabular}

was significantly up-regulated in radioresistant NPC CNE2-IR cell and which was overlap between the DEGs and the DigSee. Meanwhile, in Protein-Protein interaction network analysis, JUN was a core hub gene in PPI network. Recent research has shown that JUN is an important components of the activator protein-1 (AP-1) transcription factor and is closely related to cell proliferation, apoptosis and malignant transformation [13]. JUN could promote tumor growth and progression. Over-expression of c-jun was found to result in abnormal cell proliferation and loss of apoptosis. Some researchers have noted that inhibition the expression of c-jun can enhances radiosensitivity, induces cell cycle arrest and apoptosis [14]. Our previous study shown that the expression of c-jun was significantly up-regulated in CNE-2R cells, which may be associated with the radioresistance of NPC [15]. The kegg pathway analysis data indicated that JUN is involved in a variety of pathways. Such as TNF signaling pathway, Epstein-Barr virus infection, MAPK signaling pathway, Pathways in cancer. MAPK signaling activity confers inherent radioresistance to KRAS-mutant colorectal carcinoma cells by rapidly upregulating of heterogeneous 

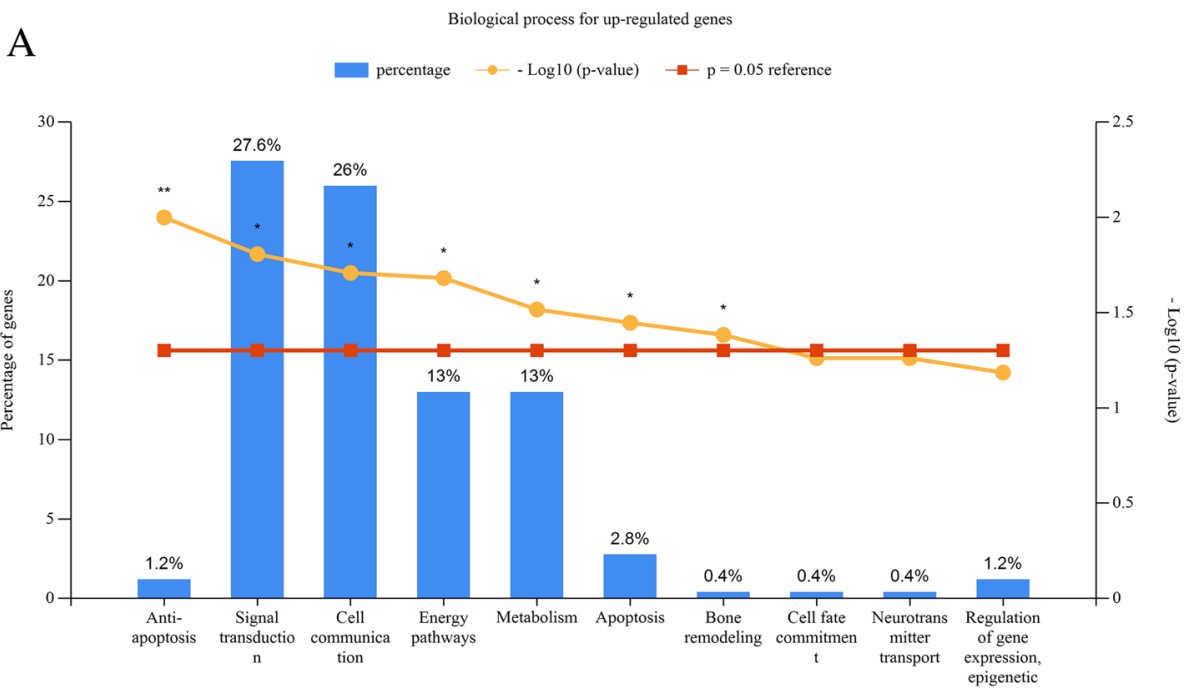

Biological process


Fig. $2 \mathrm{GO}$ functional annotation of DEGs. a The top 20 significant biology process of up-regulated genes. $\mathbf{b}$ The significant biology process of down-regulated genes

nuclear ribonucleoprotein $\mathrm{K}$ (hnRNPK) [16]. Our result showed that JUN was involved in Hepatitis B, NOD-like receptor signaling pathway, AGE-RAGE signaling pathway in diabetic complications, Pathways in cancer, IL-17 signaling pathway, TNF signaling pathway (Fig. 3b). We speculated that JUN may be participated in the regulation of nasopharyngeal carcinoma radiosensitivity through these pathways, the internal mechanism may need to be further tested in the future experiments. Based on the above, we conclude that JUN is involved in NPC radioresistance, which may provide new clues to improve radiosensitivity of Nasopharyngeal Carcinoma.

microRNAs are single stranded, endogenous, 19-25 nucleotide (nt), which are thought to be modulated tumor radiosensitivity [17]. Therefore, identification of radioresistance associated miRNAs which may contribute to more effective treatments for NPC patients. In this study, we screened out 14 differentially expressed miRNAs in the radioresistant CNE2-R cells, including the up-regulated mirRNA-762, mirRNA-1202, mirRNA-193b, mirRNAlet-7e and down-regulated mirRNA-203, mirRNA-545, mirRNA-4291, mirRNA-183, mirRNA-24, mirRNA-130a, miRNA-660, miRNA-31, miRNA-30a, miRNA-23a, suggesting that the regulation of these miRNAs might be participate in the NPC radioresistance. Most of them have been shown to be associated with radioresistance of tumor $[2,18,19]$. mirRNA-let-7e and miRNA-31 have been recently discovered to involving in the acquisition of cancer 


\begin{tabular}{|c|c|c|}
\hline \multicolumn{3}{|l|}{$\mathrm{A}$} \\
\hline Kegg pathway & miRNAs & P.value \\
\hline Proteoglycans in cancer & 17 & $4.03 E-12$ \\
\hline Viral carcinogenesis & 16 & $4.05 E-10$ \\
\hline Adherens junction & 17 & $1.66 \mathrm{E}-09$ \\
\hline Protein processing in endoplasmic reticulum & 17 & $1.34 E-08$ \\
\hline Ubiquitin mediated proteolysis & 16 & $9.73 \mathrm{E}-08$ \\
\hline Hepatitis B & 15 & $1.02 E-07$ \\
\hline Spliceosome & 17 & $1.98 \mathrm{E}-07$ \\
\hline Cell cycle & 17 & $2.62 \mathrm{E}-07$ \\
\hline Lysine degradation & 15 & $3.51 \mathrm{E}-07$ \\
\hline Chronic m yeloid leukemia & 16 & $1.20 \mathrm{E}-06$ \\
\hline Pancreatic cancer & 16 & $1.33 \mathrm{E}-06$ \\
\hline mTOR signaling pathway & 13 & $1.24 \mathrm{E}-05$ \\
\hline Renal cell carcinoma & 14 & $1.59 \mathrm{E}-05$ \\
\hline Pathways in cancer & 17 & $1.59 \mathrm{E}-05$ \\
\hline Thyroid hormone signaling pathway & 15 & $1.82 \mathrm{E}-05$ \\
\hline Hippo signaling pathway & 16 & $2.38 \mathrm{E}-05$ \\
\hline Fatty acidm etabolism & 12 & $2.77 \mathrm{E}-05$ \\
\hline Endocytosis & 17 & $2.78 \mathrm{E}-05$ \\
\hline Prion diseases & 12 & $4.04 E-05$ \\
\hline RNA transport & 16 & $4.12 \mathrm{E}-05$ \\
\hline ECM-receptor interaction & 15 & $\square .91 \mathrm{E}-05$ \\
\hline Prostate cancer & 15 & $6.08 \mathrm{E}-05$ \\
\hline p53 signaling pathway & 15 & $6.09 \mathrm{E}-05$ \\
\hline Focal achesion & 17 & $6.09 \mathrm{E}-05$ \\
\hline Small cell lung cancer & 15 & $6.99 \mathrm{E}-05$ \\
\hline AMPK signaling pathway & 15 & 0.00011630 \\
\hline Oocyte meiosis & 16 & 0.00011630 \\
\hline Non-small cell lung cancer & 14 & 0.00012006 \\
\hline TGF-beta signaling pathway & 13 & 0.00024274 \\
\hline DNA replication & 16 & 0.00024685 \\
\hline
\end{tabular}

C

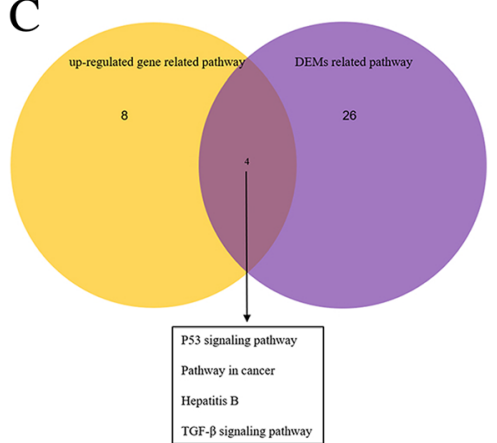

B



D

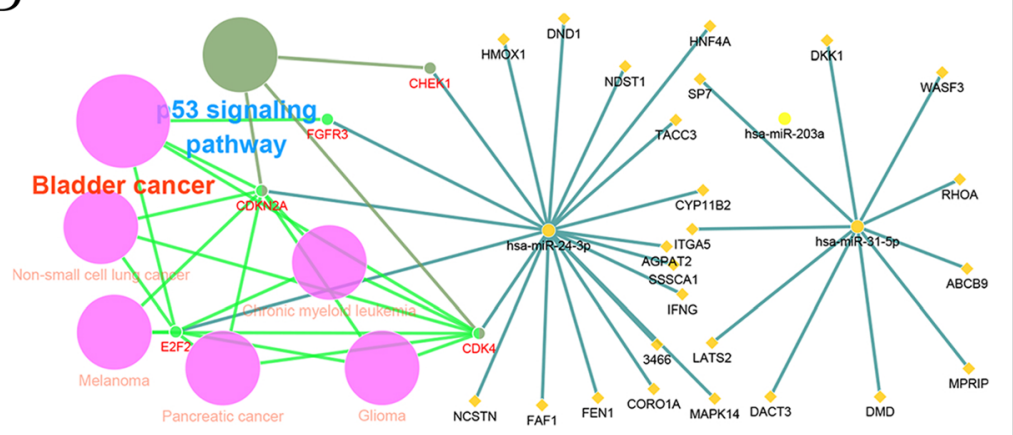

Fig. 3 Pathway enrichment analysis of DEMs target genes and DEGs. a The top 30 enriched kegg pathway for DEMs target genes. b Significant pathways in up-regulated genes. c Venn diagrams show the common pathway between upregulated genes and DEMs target genes. $\mathbf{d}$ Biomolecular network about 5 validated genes (in red) targeted by the common microRNAs and corresponding pathways were analyzed by Clue Go and Clue Pedia. The yellow diamond nodes represent target gene, the violet circle and red circle nodes represent miRNA and their related pathway respectively

cell radioresistance [1, 19]. Recent reports indicate that miRNA-23a is downregulated in the radioresistant NPC tissues, and is an independent predictor of poor prognosis in patients with nasopharyngeal carcinoma. up-regulated miRNA-23a improves NPC cell radiosensitivity in vivo and vitro. Downregulated miRNA-23a increases NPC radioresistance through activating IL-8/Stat3 signaling. Targeting miR-23a/IL-8/Stat3 signaling might be an effective approach to improving radiosensitivity of NPC [18]. It has been reported that miR-24 is frequently downregulated in NPC cell lines, and the consumption of miR-24 inhibited NPC cell growth and proliferation, while improving the radiosensitivity of NPC both in vitro and in vivo. In addition, it is reported that SP1 was verified as a target for miR-24, miR-24/SP pathway should help us understand the radiosensitivity mechanisms of human NPC, which may be a potential therapeutic target [20]. Our study detected that miRNA-24 is down-regulated in radioresistant NPC cells, and which not only was common in DEMs and JUN-related microRNAs, but also had significantly enriched in P53 signaling pathway. In conclusion, we can infer that the above mention microRNAs, especially miRNA-24 may be a key factor to affect the radiosensitivity of NPC, which may be helpful to predict radiosensitivity in NPC. 




Fig. 4 Constructed PPI network of DEGs by STRING software. Using the STRING software, proteins are represented with nodes and the interactions with continuous lines to represent direct interactions (physical), while indirect ones (functional) are presented by interrupted lines. Line thickness indicates the strength of data support

To completely understand the function of miRNAs and mRNAs in radioresistant NPC, we performed pathway enrichment analysis of DEGs and DEMs, the result demonstrated that 4 pathways are considered as the key pathways for the radiosensitivity of NPC, including TGF-beta signaling pathway, Hepatitis B, Pathways in cancer and p53 signaling pathway, which were regulated by miRNA and mRNA together. Moreover, hsa-miR-24 and its target gene were found have significantly enriched in P53 signaling pathway. P53 can predict cancer response to IR and 


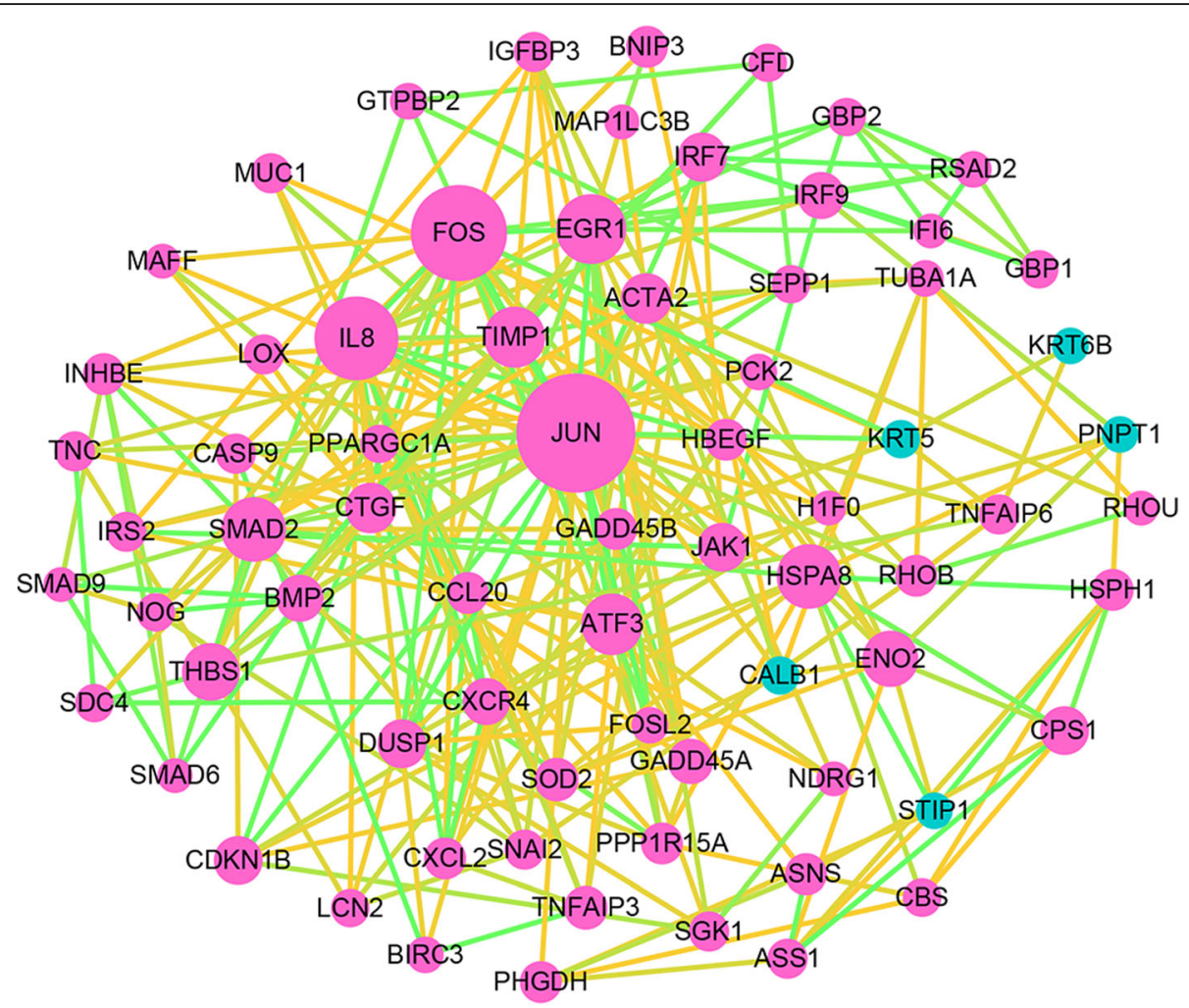

Fig. 5 Significant subnetwork of DEGs. Red nodes represent up-regulated genes, while Green nodes denote down-regulated genes. The size of the nodes is positively correlated with the count of genes. The color of line is determined by the combined score provided by STRING

chemotherapy [21]. Improving the radiosensitivity of nonsmall cell lung cancer cells by inhibition of TGF- $\beta 1$ signaling [22]. It is reported that p53 signaling pathway correlates with the radioresponse of non-small cell lung cancer. Differentially expressed genes in the p53 signaling pathway related to DNA damage repair, apoptosis, cycle regulation, metastasis, deterioration and radioresistance [23]. Previous study has shown that p53 signaling pathway mediate inhibition and apoptosis induced by $12 \mathrm{C} 6+$ heavy ion beam irradiation on HepG2 cancer cells [24]. Accordingly, we can infer that hsa-miR-24 and p53 signaling pathway should provide an important contribution to understand the mechanisms of radiosensitivity in human NPC and that it may represent a potential therapeutic target.

\section{Conclusion}

In conclusion, this study demonstrates that the upregulated gene JUN was overlap both in DEGs and publicly available studies, was a core hub gene in PPI network, which was potentially targeted by three miRNAs, including hsa-miR-203, hsa-miR-24 and hsa-miR-31. Pathway analysis showed that both up-regulated gene and DEMs were enriched in TGF-beta signaling pathway, Hepatitis B, Pathways in cancer and p53 signaling pathway. Finally, we analyzed the biological networks for validated target gene of common
miRNAs, the result indicated that miR-24 is frequently down-regulated in radioresistant NPC cell lines and significantly enriched in P53 signaling pathway. Based on these reasons, our study indicated that the JUN, miR-24 and P53 signaling pathway may be associated with radioresistance in Nasopharyngeal Carcinoma, and which may provide new clues for improving radiosensitivity in Nasopharyngeal Carcinoma. However, these results are only speculated by the combination of databases and bioinformatics methods, and still needs to be confirmed. In the following study, we explored the association between JUN expression and radioresistance in vitro. To further determine the clinical predictive value of JUN in NPC, we performed immunohistochemistry assays to examine the protein expression pattern of JUN in NPC specimens and normal nasopharyngeal epithelium specimens. And then, we performed a log-rank test analysis the Overall survival (OS) of patients with NPC based on JUN expression. Finally, Study the correlation between JUN expression and distant metastasis in patients with NPC.

\section{Methods}

\section{Microarray data}

The mRNA expression profile of GSE48501 and microRNA profile of GSE48502 were downloaded from the GEO database (Gene expression omnibus, http://www.ncbi.nlm.nih. 
gov/geo/) [7] (Additional file 1). As previousy described, the raw data was preprocessed by the application of bioconductor package 'affy' [6]. The sample of GSE48501 included 2 radioresistant NPC samples and 2 radiosensitive NPC in total, whereas the GSE48502 miRNA expression profiles included 3 radioresistant NPC samples and 3 radiosensitive NPC samples in total.

\section{Identification of DEGs and DEMs}

GEO2R (http://www.ncbi.nlm.nih.gov/geo/geo2r/) is a web tool that can analyze almost GEO series. DEGs and DEMs were screened with GEO2R [25] (Additional file 1). The differentially expressed mRNAs were selected using adjusted $P$ -values $<0.05$ and $|\operatorname{logFC}| \geq 1 . P<0.05$ and fold-change $\geq 1.5$ were set as the cut-off criterion in the DEMs. Furthermore, we applied to the online tool Morpheus (https://software. broadinstitute.org/morpheus/) to generate a heat map of DEGs [26] (Additional file 1).

\section{Screening common genes between the DEGs and the publicly available studies by Venn diagram}

We applied to disease gene search engine with evidence sentence (http://210.107.182.61/geneSearch/) to identify the radioresistant related genes for publicly available studies using the following keywords: "radioresistance" [9]. Then, the overlapping genes between the DEGs and the DigSee were screened by Venn diagram (http://bioinformatics.psb.ugent.be/webtools/Venn/) [27] (Additional file 1).

\section{Gene ontology and pathway analysis of DEGs and DEMs target genes}

Gene ontology (GO) biological process terms and pathway enrichment analysis of differentially expressed genes was performed using FunRich software (www.funrich.org/) and Clue Go software respectively $[10,28]$ (Additional file 1). The $P$-value $<0.05$ was considered significant $G O$ and pathway term. DIANA miRPATH tool (http://www.funrich.org/) was used to analyze gene ontology and pathway analysis of DEMs target genes [29] (Additional file 1). $P$-value $<0.05$ was set as the cut-off criterion in the significant GO terms and kegg pathway.

\section{PPI network construction and subnetwork mining}

The STRING database (http://string-db.org/) is an online tool to construct protein-protein interaction network [30] (Additional file 1). The Cytoscape software is a tool for the visual exploration of interaction networks composed of protein, gene, and other types of interactions [31] (Additional file 1). In present study, the protein-protein interaction network (PPI) of DEGs was mapped by STRING and then visualized using Cytoscape. Combined score $\geq$ 0.4 was set as the cut-off criterion. The proteins with high degrees were considered as the hub nodes. In addition, we further constructed subnetwork mining in the PPI network based on CentiScape with centrality value is high/ equals threshold 5.

\section{Hub genes related microRNAs were predicted using mirDIP online software}

mirDIP online software (http://ophid.utoronto.ca/mirDIP/ index.jsp) integrates twelve microRNA prediction datasets from six different microRNA prediction databases [32] (Additional file 1). In present study, hub genes corresponding microRNAs were predicted by mirDIP software. Top 5\% was named high, Score class = high was set as the cut-off criterion.

\section{Analysis of biological networks for common miRNAs}

Firstly, we used venn diagram to screen common microRNAs between JUN related microRNAs and DEMs. Then we established a regulatory network for common miRNAs, their target genes and pathways by Clue Go and Clue Pedia [1]. $P$-value $\leq 0.05$, cluster $\geq 3$ and $\min$ genes $\geq 4 \%$ was named significant biological networks (Additional file 1).

\section{Additional file}

Additional file 1: Related data in this article. (PDF $77 \mathrm{~kb}$ )

\section{Abbreviations}

DEGs: Differentially expressed genes; DEMs: Differentially expressed microRNAs; GEO: Gene expression omnibus; GO: gene ontology; NPC: nasopharyngeal carcinoma; PPI: Protein-protein interaction network

\section{Acknowledgements}

The authors will thank Ya Li Wang for her great help in bioinformatics. Thanks Xiao Z, Li X, Qu J for providing analyzable data for this study, including GSE48501and GSE48502 which can be downloaded from the GEO database.

Funding

The publication cost of this article was funded by Shaanxi Provincial Natural Science Foundation [grant numbers 2017JQ8057].

\section{Availability of data and materials}

All data generated or analyzed during this study are included in this published article [and its supplementary information files].

\section{Authors' contributions}

$G Y$ wrote the main manuscript text and designed the experiment. M Y N and HY prepared Figs. 1, 2 and 3. Z Y and Z S J prepared Figs. 4 and 5. All authors read and approved the final manuscript.

Ethics approval and consent to participate Not applicable.

\section{Consent for publication}

Not applicable.

\section{Competing interests}

The authors declare that they have no competing interests.

\section{Publisher's Note}

Springer Nature remains neutral with regard to jurisdictional claims in published maps and institutional affiliations. 


\section{Author details}

'Department of Oncology, The Second Affiliated Hospital of Medical College, Xi'an Jiao Tong University, 157 xi wu road, Xi'an 710004, People's Republic of China. ${ }^{2}$ Department of Oncology, Kashi No.2 peoples' Hospital of Xin Jiang, Kashi 844000, Xin jiang, China.

Received: 3 August 2018 Accepted: 22 April 2019

Published online: 28 May 2019

\section{References}

1. Li G, Liu Y, Liu C, Su Z, Ren S, Wang Y, Deng T, Huang D, Tian Y, Qiu Y. Genome-wide analyses of long noncoding RNA expression profiles correlated with radioresistance in nasopharyngeal carcinoma via nextgeneration deep sequencing. BMC Cancer. 2016;16:719.

2. Li G, Qiu Y, Su Z, Ren S, Liu C, Tian Y, Liu Y. Genome-wide analyses of radioresistance-associated miRNA expression profile in nasopharyngeal carcinoma using next generation deep sequencing. PLoS One. 2013;8(12):e84486,

3. Lin H, Chen Z, Zhu X, Li L, Qu S, Wei Z, Su F, Wei J, Liang Z, Mo Q, et al. Serum CD166: a novel biomarker for predicting nasopharyngeal carcinoma response to radiotherapy. Oncotarget. 2017:8(38):62858-67.

4. Fan L, Yu X, Huang Z, Zheng S, Zhou Y, Lv H, Zeng Y, Xu J, Zhu X, Yi X. Analysis of microarray-identified genes and MicroRNAs associated with idiopathic pulmonary fibrosis. Mediat Inflamm. 2017;2017:1804240.

5. Barrett T, Wilhite S, Ledoux P, Evangelista C, Kim I, Tomashevsky M, Marshall K, Phillippy K, Sherman P, Holko M, et al. NCBI GEO: archive for functional genomics data sets--update. Nucleic Acids Res. 2013; 41(Database issue):D991-5.

6. Mou T, Zhu D, Wei X, Li T, Zheng D, Pu J, Guo Z, Wu Z. Identification and interaction analysis of key genes and microRNAs in hepatocellular carcinoma by bioinformatics analysis. World I Surg Oncol. 2017;15(1):63.

7. Li X, Qu J, Yi H, Zhang P, Yi H, Wan X, He Q, Ye X, Yuan L, Zhu J, et al. Integrated analysis of differential miRNA and mRNA expression profiles in human radioresistant and radiosensitive nasopharyngeal carcinoma cells. PLoS One. 2014;9(1):e87767.

8. Yang Z, Zhuan B, Yan Y, Jiang S, Wang T. Identification of gene markers in the development of smoking-induced lung cancer. Gene. 2016;576(1 Pt 3):451-7.

9. Wang $Y$, Yin W, Zhu X. Blocked autophagy enhances radiosensitivity of nasopharyngeal carcinoma cell line CNE-2 in vitro. Acta Otolaryngol. 2014; 134(1):105-10

10. Gao H, Wang H, Yang W. Identification of key genes and construction of microRNAmRNA regulatory networks in multiple myeloma by integrated multiple GEO datasets using bioinformatics analysis. Int J Hematol. 2017;106(1):99-107.

11. Chen W, Hu G. Biomarkers for enhancing the radiosensitivity of nasopharyngeal carcinoma. Cancer Biol Med. 2015;12(1):23-32.

12. Li S, Liu T, Mo W, Hou Q, Zhou Y, Liu M, He Z, Liu Z, Chen Q, Wang H, et al. Prognostic value of phosphorylated Raf kinase inhibitory protein at serine 153 and its predictive effect on the clinical response to radiotherapy in nasopharyngeal carcinoma. Radiat Oncol. 2016;11(1):121.

13. Shaulian E. AP-1--the Jun proteins: oncogenes or tumor suppressors in disguise? Cell Signal. 2010;22(6):894-9.

14. Qing H, Gong W, Che Y, Wang X, Peng L, Liang Y, Wang W, Deng Q, Zhang $\mathrm{H}$, Jiang B. PAK1-dependent MAPK pathway activation is required for colorectal cancer cell proliferation. Tumour Biol. 2012;33(4):985-94.

15. Guo Y, Zhu X, Qu S, Li L, Su F, Li Y, Huang S, Li D. Identification of genes involved in radioresistance of nasopharyngeal carcinoma by integrating gene ontology and protein-protein interaction networks. Int J Oncol. 2012;40(1):85-92.

16. Eder S, Arndt A, Lamkowski A, Daskalaki W, Rump A, Priller M, Genze F, Wardelmann E, Port M, Steinestel K. Baseline MAPK signaling activity confers intrinsic radioresistance to KRAS-mutant colorectal carcinoma cells by rapid upregulation of heterogeneous nuclear ribonucleoprotein K (hnRNP K). Cancer Lett. 2017;385:160-7.

17. Song Y, Zuo Y, Qian X, Chen Z, Wang S, Song L, Peng L. Inhibition of MicroRNA$21-5 p$ promotes the radiation sensitivity of non-small cell lung Cancer through HMSH2. Cell Physiol Biochem. 2017;43(3):1258-72.

18. Qu J, Yi H, Ye X, Li L, Zhu J, Xiao T, Yuan L, Li J, Wang Y, Feng J, et al. MiR23a sensitizes nasopharyngeal carcinoma to irradiation by targeting IL-8/ Stat3 pathway. Oncotarget. 2015;6(29):28341-56.

19. Lynam-Lennon N, Reynolds J, Marignol L, Sheils O, Pidgeon G, Maher S. MicroRNA-31 modulates tumour sensitivity to radiation in oesophageal adenocarcinoma. J Mol Med. 2012;90(12):1449-58.
20. Kang M, Xiao J, Wang J, Zhou P, Wei T, Zhao T, Wang R. MiR-24 enhances radiosensitivity in nasopharyngeal carcinoma by targeting SP1. Cancer Med. 2016;5(6):1163-73.

21. Rashi-Elkeles S, Elkon R, Shavit S, Lerenthal Y, Linhart C, Kupershtein A, Amariglio N, Rechavi G, Shamir R, Shiloh Y. Transcriptional modulation induced by ionizing radiation: p53 remains a central player. Mol Oncol. 2011;5(4):336-48.

22. Zhao $Y$, Wang L, Huang $Q$, Jiang $Y$, Wang J, Zhang L, Tian $Y$, Yang $H$. Radiosensitization of non-small cell lung Cancer cells by inhibition of TGF- $\beta 1$ signaling with SB431542 is dependent on p53 status. Oncol Res. 2016;24(1):1-7.

23. Jung I, Kang H, Kim K, Kim I. PTEN/pAkt/p53 signaling pathway correlates with the radioresponse of non-small cell lung cancer. Int J Mol Med. 2010; 25(4):517-23.

24. Liu K, Zhao X, Gu J, Wu J, Zhang H, Li Y. Effects of 12C6+ heavy ion beam irradiation on the p53 signaling pathway in HepG2 liver cancer cells. Acta Biochim Biophys Sin Shanghai. 2017;49(11):989-98.

25. Qi Y, Chen X, Wu N, Ma C, Cui X, Liu Z. Identification of risk factors for sepsisassociated mortality by gene expression profiling analysis. Mol Med Rep. 2018.

26. Mi B, Liu G, Zhou W, LV H, Liu Y, Liu J. Identification of genes and pathways in the synovia of women with osteoarthritis by bioinformatics analysis. Mol Med Rep. 2018

27. Niu Y, Hu B, Li X, Chen H, Takáč T, Šamaj J, Xu C. Comparative digital gene expression analysis of tissue-cultured plantlets of highly resistant and susceptible Banana Cultivarsin response to Fusarium oxysporum. Int J Mol Sci. 2018;19(2)

28. Chen Z, Wu H, Wang G, Feng Y. Identification of potential candidate genes for hypertensive nephropathy based on gene expression profile. BMC Nephrol. 2016;17(1):149.

29. Wu A, Lou L, Zhai J, Zhang D, Chai L, Nie B, Zhu H, Gao Y, Shang H, Zhao M: miRNA expression profile and effect of Wenxin granule in rats with ligation-induced myocardial infarction. Int J Genomics 2017, 2017:2175871.

30. Szklarczyk D, Franceschini A, Wyder S, Forslund K, Heller D, Huerta-Cepas J, Simonovic M, Roth A, Santos A, Tsafou K, et al. STRING v10: protein-protein interaction networks, integrated over the tree of life. Nucleic Acids Res. 2015;43(Database issue):D447-52.

31. Wang $Y$, Zhao $Q$, Lan N, Wang S. Identification of methylated genes and miRNA signatures in nasopharyngeal carcinoma by bioinformatics analysis. Mol Med Rep. 2018.

32. Tokar T, Pastrello C, Rossos A, Abovsky M, Hauschild A, Tsay M, Lu R, Jurisica I. mirDIP 4.1-integrative database of human microRNA target predictions. Nucleic Acids Res. 2018;46(D1):D360-70

Ready to submit your research? Choose BMC and benefit from:

- fast, convenient online submission

- thorough peer review by experienced researchers in your field

- rapid publication on acceptance

- support for research data, including large and complex data types

- gold Open Access which fosters wider collaboration and increased citations

- maximum visibility for your research: over $100 \mathrm{M}$ website views per year

At $\mathrm{BMC}$, research is always in progress.

Learn more biomedcentral.com/submissions 